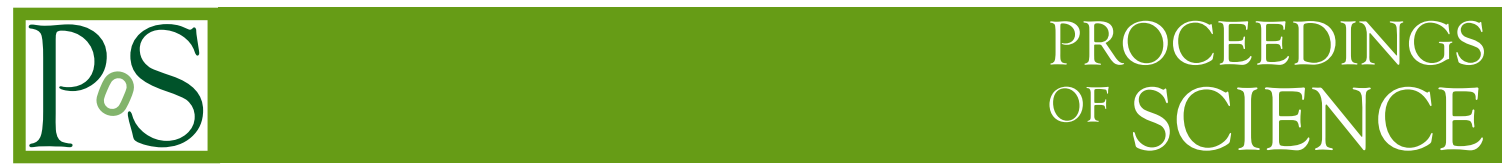

\title{
Large order behavior of Wilson loops from NSPT
}

\author{
Ernst-Michael Ilgenfritz ${ }^{* \dagger}$ \\ Veksler and Baldin LHEP, JINR, Dubna 141980 Russia \\ E-mail: ilgenfri@lhep.jinr.ru
}

Following numerical stochastic perturbation theory, we have performed Langevin simulation of Wilson loops [1]. This allows us insight into perturbation theory for Wilson loops to very high order. Thus, we are able to exclude a factorial rise of the coefficients of the expansion in $g^{2}$. We propose a model parametrization in terms of the hypergeometric function ${ }_{2} F_{1}$ to facilitate the summation (within some radius of convergence). We discuss the possibilities to speed up the convergence of the series within boosted perturbation theory. Together with results from standard Monte Carlo simulation, the detailed knowledge of perturbation theory enables us to assess the nonperturbative part of Wilson loops and to estimate the gluon condensate.

Xth Quark Confinement and the Hadron Spectrum,

October 8-12, 2012

TUM Campus Garching, Munich, Germany

\footnotetext{
${ }^{*}$ Speaker.

${ }^{\dagger}$ for the complete list of contributing authors, see [1].
} 
What is the nonperturbative part of an observable, if it has a perturbative expansion like $\mathscr{O}_{P T} \sim \sum_{n} c_{n} g^{2 n}$ ? One possible answer is $\mathscr{O}_{N P}=\mathscr{O}_{M C}-\mathscr{O}_{P T}$. When the convergence is - mostly asymptotic, the question remains to be answered: At which order should one truncate a perturbative series in $g^{2}$ ? Narison and Zakharov [2] have discussed the impact of choosing between short and long perturbative series on the determination of the gluon condensate(s). How can one get a global view of the behavior of a perturbative series, say, for Wilson loops ? Numerical Stochastic Perturbation Theory (NSPT) [3, 4] allows to address this problem.

First studies using NSPT up to 8-th order [5] and up to 10-th order [6] have been analyzed following the renormalon paradigm assuming that the expansion coefficients can be modelled by a renormalon ansatz, $c_{n} \sim C_{1}\left(C_{2}\right)^{n} \Gamma\left(n+C_{3}\right)$. A reanalysis of the 10-th order results [7] gave arguments against the renormalon scenario, finally also against a non-vanishing 2-nd order gluon condensate. Now, our paper [1] describes the perturbative behavior of Wilson loops up to 20-th order, confirming the results of [7] and allowing a model for summing the series, however with a finite radius of convergence.

NSPT [3, 4] is based on Langevin simulation, in the case at hand for the Wilson action. It realizes a stochastic evolution of the links of the lattice (backed by stochastic gauge fixing) :

$$
\frac{\partial}{\partial \tau} U_{x, \mu}(\tau ; \eta)=\mathrm{i}\left\{\nabla_{x, \mu} S_{W}[U]-\eta_{x, \mu}(\tau)\right\} U_{x, \mu}(\tau ; \eta)
$$

with the force $F_{x, \mu}[U, \eta]=\varepsilon \nabla_{x, \mu} S_{W}[U]+\sqrt{\varepsilon} \eta_{x, \mu}$ corresponding to the action $S_{W}$. One has to take the limit of vanishing time step $\varepsilon$ in all observables obtained by Langevin averaging. Consider the links and the force expanded in orders of $g$, say, $U_{x, \mu}=1+\sum_{m>0} U_{x, \mu}^{(m)} g^{m}$. Then a hierarchy of Langevin updates results, where the white noise $\eta$ enters through the lowest order $F^{(1)}$ :

$$
\begin{aligned}
& U^{(1)}(n+1)=U^{(1)}(n)-F^{(1)}(n) \\
& U^{(2)}(n+1)=U^{(2)}(n)-F^{(2)}(n)+\frac{1}{2}\left(F^{(1)}(n)\right)^{2}-F^{(1)}(n) U^{(1)}(n) \text { etc. }
\end{aligned}
$$

From the expansion of links one gets the expansion of Wilson loops of rectangular shape $N \times M$ [1]

$$
W_{N M}[U]=\sum_{n=0,1 / 2,1,3 / 2, \ldots} W_{N M}^{(n)} g^{2 n}=\frac{1}{3} \operatorname{Tr} \prod_{(x, \mu) \in(N \times M)}\left[\sum_{m_{x, \mu} \geq 0} U_{x, \mu}^{\left(m_{x, \mu}\right)} g^{m_{x, \mu}}\right] .
$$

The Domb-Sykes plot [8] characterizes the large- $n$ behavior of a series $\sum_{n} c_{n} g^{2 n}$, showing the ratio of subsequent coefficients $r_{n}=c_{n} / c_{n-1}$ as a function of $1 / n$. A straight line signals a powerlike singularity $\left(1-u g^{2}\right)^{\gamma}$ with the radius of convergence $1 / u: r_{n}=u(1-(1+\gamma) / n)$. A small curvature can be accounted for by the modification : $(1+\gamma) / n \rightarrow(1+\gamma) /(n+s)$. Horsley et al. [7] (based on 10 orders of NSPT [6]) had fitted the plaquette with $u=0.961(9), \gamma=0.99(7)$ and $s=0.44(10)$ (see Fig. 1 left).

Now, based on 20 orders accessible by NSPT [1], we found the fit for small loops $W_{N M}$ :

$$
r_{n}=\frac{c_{n}}{c_{n-1}}=u\left(1-\frac{1+\gamma}{n}\right)+\frac{p}{n(n+s)}
$$

This result can be recursively used for $n>n_{0}$, starting from any numerically found $c_{n_{0}}$. All this can 

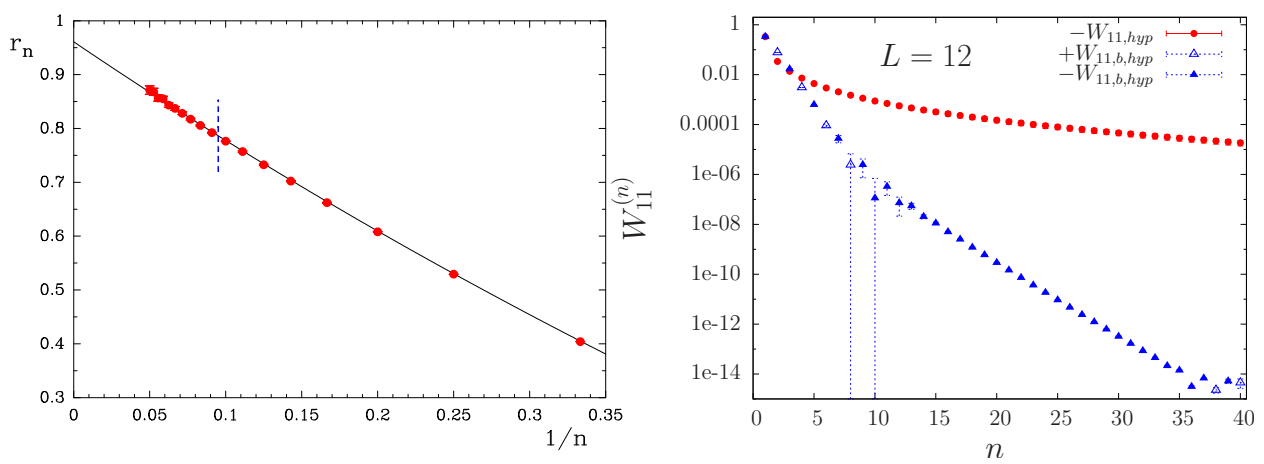

Figure 1: Left: Domb-Sykes plot for the plaquette, compared with the prediction of 2001 [7], based on data for $n \leq 10$. Right: Comparison of the coefficients of the naive (red) and the boosted series (blue) for $W_{11}$.

be summarized by a "Hypergeometrical Model" for $W_{11}, W_{21}, W_{22}$ and $W_{31}[1]$

$$
W_{N M, \infty}^{\left(n_{0}\right)}=1+\sum_{n=1}^{n_{0}}\left(c_{n}-d_{n_{0}} A_{n} u^{n}\right) g^{2 n}+d_{n_{0}}\left[{ }_{2} F_{1}\left(\sigma-\tau-1, \sigma+\tau-1 ; s+1 ; u g^{2}\right)-1\right]
$$

with a common inverse convergence radius $u=0.9694\left(\beta_{c} \approx 5.82\right)$. Here are $\sigma=(s+3-\gamma) / 2$ and $\tau=\sqrt{(\gamma+s+1)^{2}-4 p / u} / 2$ entering the arguments.

Boosted perturbation theory can speed up the convergence. $g_{b}^{2}=g^{2} / W_{11}\left(g, n^{*}\right)$ is the boosted coupling defined in terms of the truncated perturbative plaquette $W_{11}\left(g, n^{*}\right)=1+\sum_{n=1}^{n^{*}} W_{11}^{(n)} g^{2 n}$. The sum $W_{11}\left(g, n^{*}\right)$ is called "naive series" truncated at $n^{*}$. Then $W_{N M, b}\left(g_{b}, n^{*}\right)=1+\sum_{n=1}^{n^{*}} W_{N M, b}^{(n)} g_{b}^{2 n}$ is called "boosted series" truncated at $n^{*}$. The latter converges much more rapidly, although the boosted coupling $g_{b}$ is larger than $g$. The coefficients $W_{N M, b}^{(n)}$ of the boosted series are calculated by equating $W_{N M}\left(g, n^{*}\right)=W_{N M, b}\left(g_{b}, n^{*}\right)$.

A precise separation of the non-perturbative part of Wilson loops requires a very high order of PT. We have employed boosting using the hypergeometric model to smooth the input obtained from NSPT and to get coefficients beyond $n^{*}=20$. The boosted coupling is computed equating (smoothed) series up to $n^{*}=40$. Fig. 1 (right) shows the corresponding coefficients for $W_{11}$ for the bare and boosted coupling.

For the special case of the plaquette the relation to the gluon condensate $\langle(\alpha / \pi) G G\rangle$ is

$$
a^{4} \frac{\pi^{2}}{36}\left(\frac{-b_{0} g^{3}}{\beta(g)}\right)\left\langle\frac{\alpha}{\pi} G G\right\rangle=W_{11, b}\left(g_{b}, n^{*}\right)-W_{11, M C} \equiv \Delta_{W_{11}}
$$

up to eventual $\mathscr{O}\left(a^{2}\right)$ or $\mathscr{O}\left(a^{8}\right)$ contributions (see Figure 2 left). We obtain for the quartic gluon condensate

$$
\left\langle\frac{\alpha}{\pi} G G\right\rangle=0.028(3) \mathrm{GeV}^{4}
$$

to be compared with the SVZ value of $0.012 \mathrm{GeV}^{4}$. Our number agrees within errors with the estimate $0.024(8) \mathrm{GeV}^{4}$ based on a study of heavy quarkonia mass splittings [9]. For full information the original paper [1] should be consulted. 

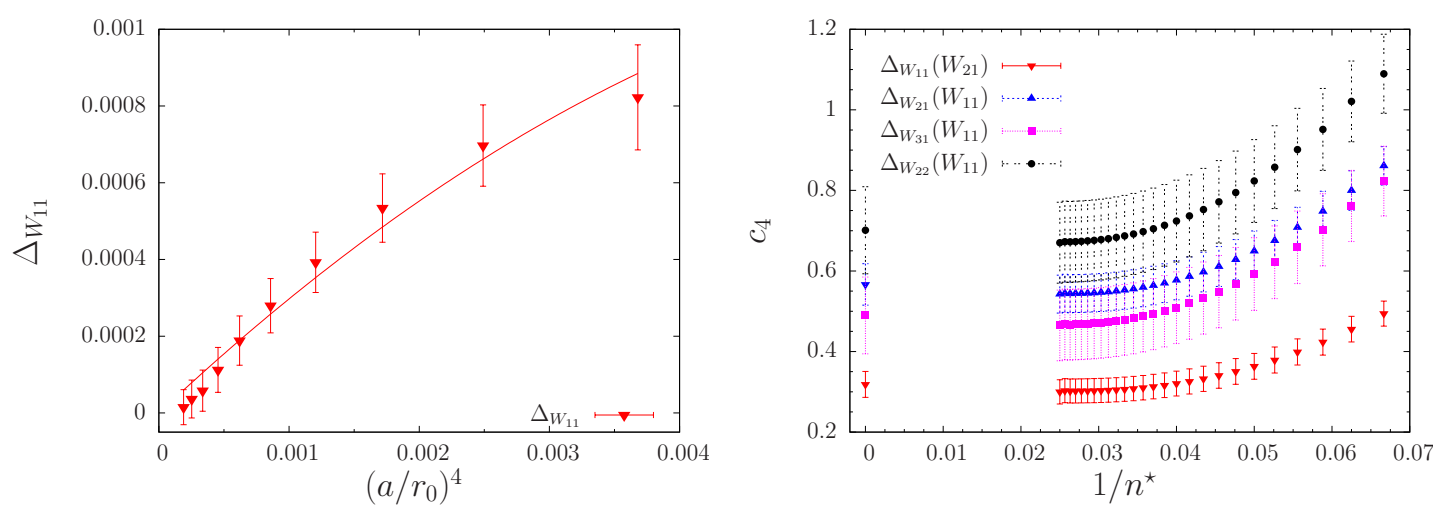

Figure 2: Left: $\Delta_{W_{11}}$ (parametrized as $\Delta_{W_{11}}=c_{2} a^{2}+c_{4} a^{4}$ ) as function of $a^{4}$. There is not much room for an $A^{2}$ condensate. Right: The coefficients $c_{4}\left(n^{*}\right)$ for different Wilson loops as functions of $1 / n^{*}$. The plateau reached for $n^{*}>30$ agrees well with the limit from the hypergeometric model $\left(1 / n^{*}=0\right)$.

\section{References}

[1] R. Horsley, G. Hotzel, E.-M. Ilgenfritz, R. Millo, H. Perlt, P.E.L. Rakow, Y. Nakamura, G. Schierholz, and A. Schiller. Phys.Rev. D86, 054502 (2012), arXiv: 1205.1659.

[2] S. Narison and V. Zakharov. Phys. Lett. B679, 355-361 (2009), arXiv:0906. 4312.

[3] F. Di Renzo, E. Onofri, G. Marchesini, and P. Marenzoni. Nucl. Phys. B426, 675-685 (1994), hep-lat/9405019.

[4] F. Di Renzo and L. Scorzato. JHEP 0410, 073 (2004), hep-lat / 0410010.

[5] G. Burgio, F. Di Renzo, G. Marchesini, and E. Onofri. Phys. Lett. B422, 219-226 (1998), hep-ph/9706209.

[6] F. Di Renzo and L. Scorzato. JHEP 0110, 038 (2001), hep-lat/ 0011067.

[7] R. Horsley, P. E. Rakow, and G. Schierholz. Nucl. Phys. Proc. Suppl. 106, 870-872 (2002), hep-lat/0110210.

[8] C. Domb and M. F. Sykes. Proc. Royal Soc. A 240, 214 (1957).

[9] S. Narison, Phys. Lett. B387 162 (1996), arXiv : hep-ph/9512348. 